\title{
Spanish-Speaking Therapists Increasingly Switch to Telepsychology During COVID-19: Networked Virtual Reality May Be Next
}

Mariana Sampaio, MSW, ${ }_{1}^{1,2}$ Maria Vicenta Navarro Haro, $\mathrm{PhD}_{1}^{3,4}$ Chelsey Wilks, PhD, ${ }^{5}$ Bruno De Sousa, PhD, ${ }^{6}$

Azucena Garcia-Palacios, $P h D_{,}^{7,8}$

and Hunter G. Hoffman, $P h D^{9-13, i}$

${ }^{1}$ Department of Psychology, University of Coimbra, Coimbra, Portugal.

${ }^{2}$ Department of Social work, Catholic University of Portugal, Lisbon, Portugal.

${ }^{3}$ Instituto Aragonés de Investigaciones Sanitarias, Universidad de Zaragoza, Zaragoza, Spain.

${ }^{4}$ Department of Psychology and Sociology, Faculty of Economics and Business, University of Zaragoza, Zaragoza, Spain.

${ }^{5}$ Department of Psychological Sciences, University of Missouri-St. Louis, St. Louis, Missouri, USA.

${ }^{6}$ Faculty of Psychology and Educational Sciences, CINEICC University of Coimbra, Coimbra, Portugal.

${ }^{7}$ CIBER Fisiopatología Obesidad y Nutrición (CIBERObn), Instituto Carlos III, Madrid, Spain.

${ }^{8}$ Department of Basic and Clinical Psychology, and Psychobiology, Universitat Jaume I, Castellón, Spain.

Departments of ${ }^{9}$ Mechanical Engineering, ${ }^{10}$ Radiology, and

${ }^{11}$ Psychology, University of Washington, Seattle, Washington, USA.

${ }^{12}$ Human Photonics Lab, Virtual Reality Research Center, University of Washington, Seattle, Washington, USA.

${ }^{13}$ Computer Science, King Abdulaziz University, Jeddah, Saudi Arabia.

iORCID ID (https://orcid.org/0000-0002-6934-3568).

\section{Abstract}

Background: Social distancing restrictions imposed due to the Novel Coronavirus 2019 (COVID-19) pandemic resulted in a rapid shift in the delivery of psychological interventions from in-person to telehealth. Much of the research on this transition has been conducted with English-speaking mental health providers, leaving a gap in understanding related to how this shift has impacted Spanish-speaking treatment providers.

Methods: Fifty non-U.S. Spanish-speaking therapists completed a survey related to their use of telecommunication modalities; client population characteristics; professional, ethical, and legal/regulatory issues; and telehealth training and practice. Participants completed the survey at one time point and retrospectively described their use of telehealth both pre-pandemic and during the pandemic.

Results: Most of the 50 Spanish-speaking therapists surveyed reported using telepsychology 58\% before COVID-19 versus $84 \%$ during the COVID-19 pandemic $\left(\chi^{2}=5.76, \mathrm{p}<0.05\right)$. Compared with pre-pandemic, the number of hours therapists spent using telepsychology per week increased significantly for early adopter therapists (those who began using telehealth before the pandemic began) $(\mathrm{Z}=-3.18, \mathrm{p}=0.001)$ and also for late adopter therapists who only began using telehealth during the pandemic $(\mathrm{Z}=-3.74, \mathrm{p}<0.001)$. Many therapists reported equity issues. Most participants also reported ethical and regulatory concerns regarding security/confidentiality or Health Insurance Porability and Accountability Act.

Conclusions: The rapid adoption of technology to deliver therapy during COVID-19 has spurred growing pains for Spanish-speaking therapists and their underserved clients, and more research is needed to better understand and improve the therapists' adoption of these technologies with diverse patient populations.

Keywords: telehealth, telepsychology, COVID-19, virtual reality, underserved, Spanish-speaking therapists

\section{Introduction}

he Novel Coronavirus 2019 (COVID-19) pandemic prompted mental health providers to shift treatment delivery from face-to-face to remote telehealth. The impacts of this rapid shift have been well documented in the United States ${ }^{1-4}$ (e.g., treatment for chronic pain and psychological problems). However, much less is known about how COVID-19 has impacted the use of telepsychology in underserved Spanish-speaking countries, ${ }^{5}$ leaving a gap in our understanding of how COVID-19 impacted Spanish-speaking treatment providers use of telepsychology. 


\section{SAMPAIO ET AL.}

While traditional face-to-face therapeutic services may have been impacted by COVID-19, the implementation of new technologies may mitigate disruptions in treatment delivery. For example, immersive virtual reality (VR) is a powerful new computer technology that therapists can use to help treat their patients by being able to meet as avatars, potentially increasing acceptance/dissemination of teletherapy, increasing compliance with homework assignments, improving therapeutic outcomes, and reducing costs. ${ }^{6-12}$

During VR mindfulness, participants wear a VR helmet that blocks their view of the real world, reducing distractions, and helps draw participant's attention into the mindfulness practice session. For example, in Mindfulness RiverWorld, patients float slowly above a three-dimensional computer-generated river, while observing sights and sounds and bringing their attention back to the river when their mind wanders (with audio instructions helping train them how to practice mindfulness). Our early case studies have shown that practicing mindfulness while in immersive VR resulted in large reductions in negative emotions and a large increase in positive emotions of patients with borderline personality disorder, ${ }^{13}$ severe burn injuries, ${ }^{14}$ and a severe spinal injury patient coping with recently being paralyzed after falling several stories out of a building. ${ }^{15}$ Preliminary results from controlled studies have also shown significant reductions in anxiety in patients with generalized anxiety disorder ${ }^{16}$ after treatment with a mindfulness-based treatment including VR mindfulness.

The current survey of non-U.S. Spanish-speaking therapists compares therapists' use, training with, and perceived benefits of online therapy before versus during COVID-19. We hypothesized that there would be a significant increase in the use of telepsychology during social isolation, and we predicted that the results would also indicate areas in need of improvement in terms of therapists' knowledge about rules and regulations of using telehealth. Regarding the impact of the COVID-19 pandemic on the therapists, we predicted that Spanish-speaking therapists would report feeling significantly more burned out during the pandemic than they felt before COVID-19.

\section{Methods}

\section{PARTICIPANTS}

A sample of 50 Spanish-speaking mental health professionals (e.g., licensed clinical psychologists, social workers, mental health counselors) were recruited to complete an online survey study on their use and experience with technology. Respondents were included if they were: (a) Spanish-speaking mental health professionals in Spain and/or Latin America and (b) provided mental health care (e.g., clinical therapy or clinical counseling services, or clinical social workers).
Potential participants were informed about the nature of the survey, and 50 therapists anonymously clicked "continue to survey." To ensure anonymity, participants were not asked to disclose personal information and were assured that no personal data would be collected that could potentially identify them, such as e-mail addresses. The current investigation was approved by the institutional review board at the University of Washington, Seattle, and was preregistered at ClinicalTrials.gov Identifier: NCT04360850.

\section{PROCEDURE}

Prospective mental health professionals were recruited by invitation and were provided with a link to a secure online survey. These invitations were distributed to an unknown number of therapists via closed social media professional groups and electronic mailing lists from different boards, federations, and institutions associated with the mental health field, as well as peer-to-peer invitation. The online survey took $\sim 10-15 \mathrm{~min}$ to complete. The time frame of data collection was from April 24, 2020, to May 18, 2020. The researchers subsequently downloaded the de-identified data from www.limesurvey.org to analyze at the University of Washington, Seattle.

\section{INSTRUMENTS}

The survey used in the current study was adapted and translated into Spanish by Sampaio et al. ${ }^{1}$ who adapted from a 28-item survey by Glueckauf et al. ${ }^{17}$ The current survey focused on evaluating four key domains of using telehealth to deliver clinical services by Spanish-speaking professional psychologists before the COVID-19 pandemic: providers' use of telecommunication modalities; client population characteristics; professional, ethical, and legal/regulatory issues; and telehealth training and practice. Some questions were adapted to assess "before pandemic" versus "during pandemic" changes in clients' needs and therapists' attitudes and behavior toward using telepsychology to conduct therapy sessions, and five new questions were added regarding the therapists' self-ratings of burnout, use of mindfulness and VR devices, the therapists' interest in VR mindfulness for selftreatment to reduce the therapists stress levels, and how much they are volunteering telehealth support.

In the current study, Spanish-speaking therapists filled out the survey at one time point and were retrospectively reporting on their therapy practices before the pandemic versus during the pandemic. The survey started with a demographic section (e.g., age, gender, ethnicity), followed by 29 True False/multiple-choice questions regarding current professional status (e.g., primary profession, work setting) and 
previous education (e.g., time since graduation). Respondents were asked about their: (a) use of telepsychology before versus during the COVID-19 pandemic (e.g., "Have you been working with telehealth/telepsychology before the Novel Coronavirus pandemic started"); (b) changes in their recruitment and retention of patients before and during COVID-19; (c) types of telecommunication modalities used in clinical practice before versus during the COVID pandemic, and types of populations and settings; (d) therapists' burnout associated with delivering therapy before versus during COVID-19 (according to Freudenberger's ${ }^{18}$ definition of burnout); (e) therapists' ethical and legal/regulatory concerns related to delivery of telepsychology services; (f) therapists' views on education and training needed to use telepsychology in clinical practice; and (g) therapists interest in using immersive VR therapy (VR mindfulness) for self-treatment to help reduce the therapists stress in the future. The survey ends with an open answer section allowing professionals to comment about any other relevant information regarding the survey topics.

\section{DATA ANALYSIS STRATEGY}

Parametric and nonparametric statistics ( $t$-tests, McNemar's test, and Wilcoxon signed-rank test) were used for within- and between-subject paired and independent comparisons. IBM SPSS $^{19}$ was used to conduct data analyses.

\section{Results}

\section{SAMPLE CHARACTERISTICS}

The mean $(M)$ age of the respondents was 39.48 years (standard deviation $[\mathrm{SD}]=12.10$ years, range $=22-78$ years) from Spain and Latin America. Participants were 78\% female. Ethnic background was predominantly Hispanic/Latino (60\%), as well as Caucasian (36\%) and middle Eastern (2\%). The primary work settings were private practice (36\%), university (24\%), mental health clinic (18\%), hospital (14\%), group practice (4\%), and public health (4\%). The therapists' countries of origin were Spain (28\%), Ecuador (20\%), Mexico (20\%), Argentina (14\%), Columbia (10\%), Chile (8\%), Peru (8\%), Uruguay (6\%), and Portugal (2\%).

Most therapists had worked a number of years since obtaining their highest academic degree $(M=13.82$ years, $\mathrm{SD}=11.25$ years, range $=22-78$ years) and had worked several years since licensure $(M=12.32$ years, $\mathrm{SD}=11.71$ years).

\section{PREFERENCE AND USE OF TELEPSYCHOLOGY BEFORE VERSUS DURING COVID-19}

According to within-subject paired comparisons, the percentage of Spanish-speaking therapists using telepsychology was 58\% before COVID-19 versus $84 \%$ during the COVID-19 pandemic, a statistically significant increase during the pandemic $\left(\chi^{2}=5.76, p<0.05\right)$. Overall, the Spanish-speaking therapists surveyed reported no change in specific training about telehealth, 24\% before COVID-19 versus 24\% during COVID-19 $\left(\chi^{2}=0\right.$, not significant [NS]). Of the 50 respondents, 29 (58\%) were early adopters and 21 respondents (42\% of the Spanish-speaking therapist surveyed) only started using telehealth during the pandemic (late adopters).

As shown in Figures 1 and 2, descriptive statistics were used in an exploratory comparison of the characteristics of early adopters (29/50) versus late adopters (21/50). Early adopters showed little change in the categories of patients they treated before versus during COVID-19. As shown in Figure 1, nearly all (i.e., 95\% before COVID-19 and 95\% during COVID-19) of early adopters were treating adults, and many (25\% before COVID-19 and 25\% during COVID-19) were treating adolescents. Early adopter therapists (who were using telepsychology before COVID-19 began) reported a small decrease in treating elderly patients during COVID-19 and reported small increases in treating children, couples, and groups during COVID-19.

Late adopters showed much greater increases in the use of telehealth during COVID-19. For the survey questions shown in Figure 2, the percentage of late adopters treating adult patients increased from 19\% before COVID-19 to 80\% during COVID-19. Similarly, the percentage of late adopters treating adolescent patients increased from 10\% before COVID-19 to

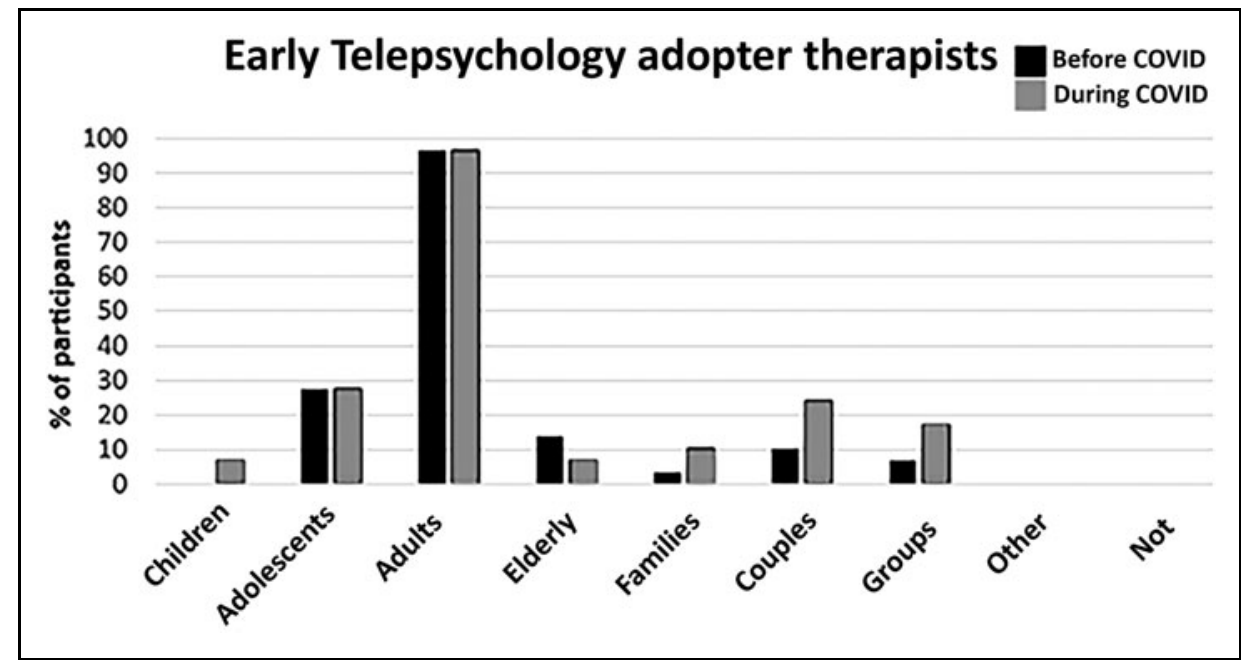

Fig. 1. Percent increase in telepsychology during pandemic (for early adopter therapists). 


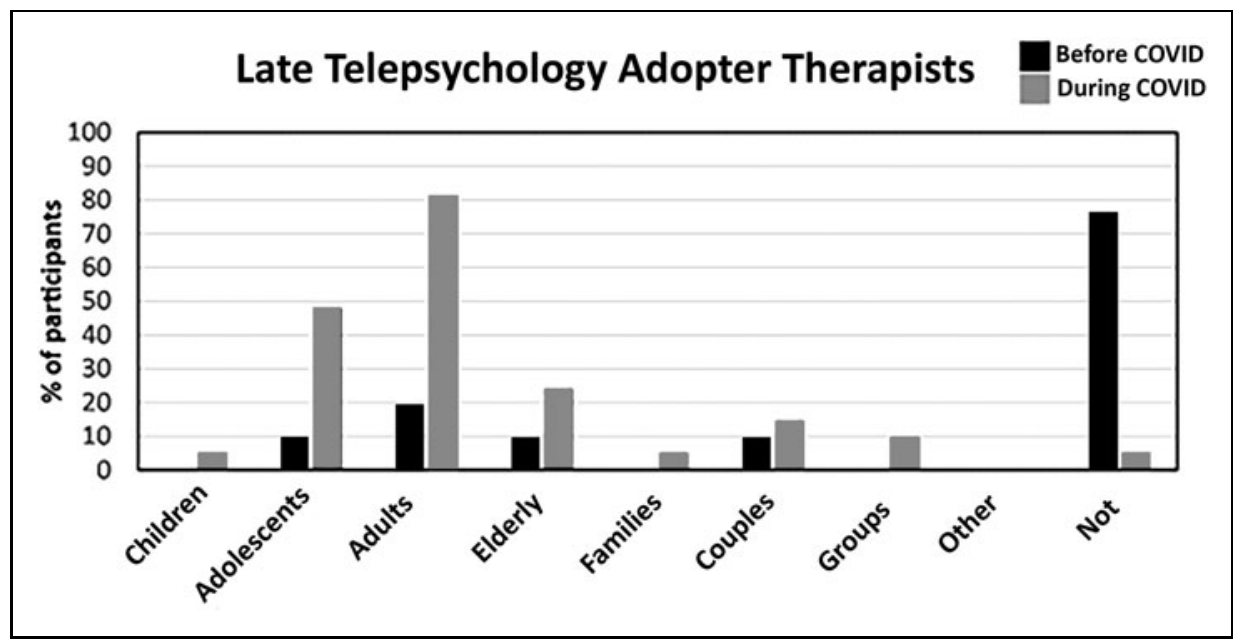

Fig. 2. Percent increase in telepsychology during pandemic (for late adopter therapists). from Skype, WhatsApp, and phone calls to predominant use of Zoom and Zoom Pro. As shown in Figure 4, late adopters showed a much different pattern of use of telepsychology technologies than early adopters. Before the pandemic, most therapists who were late to use telepsychology (i.e., late adopters) used phones, e-mails, and texts to communicate with their patients (if they used tele-technologies at all). However, during COVID-19, late adopters reported large increases in use of Skype, WhatsApp, Zoom, and phones during COVID-19. Interestingly, as shown in Table 1, 20\% of early adopters reported using VR to treat 48\% during COVID-19. Late adopter therapists showed small increases during COVID-19 in all other patient categories: children, adolescents, adults, elderly, family therapy, couples, and groups.

The survey also measured what different types of telepsychology technologies therapists were using before versus during COVID-19, for early and late adopters. As shown in Figure 3, before COVID-19, early adopter therapists were primarily using Skype, WhatsApp, personal phone calls, e-mails, and text messages to communicate with their patients before COVID-19, but the frequency of use of each of these technologies dropped during COVID-19. During COVID-19, for early adopters, the only technology that increased during COVID-19 was Zoom and Zoom Pro. So early adopters shifted their patients before COVID-19 began, and a large number of therapists (84\%) expressed interest in trying immersive VR mindfulness to reduce their own stress in the future.

Forty-four percent of the 50 therapists reported a reduction in requests from current clients and 26\% reported an increase in requests for therapy services from current clients during COVID-19, and 30\% stayed the same before versus during the pandemic. Similarly, 38\% of the 50 therapists reported a reduction in requests from new clients and 28\% reported an increase in requests for therapy services from new clients during COVID-19, and 34\% stayed the same before versus during the pandemic. As discussed next, early and late adopters ended up spending the same number of hours per week using telehealth during the pandemic.

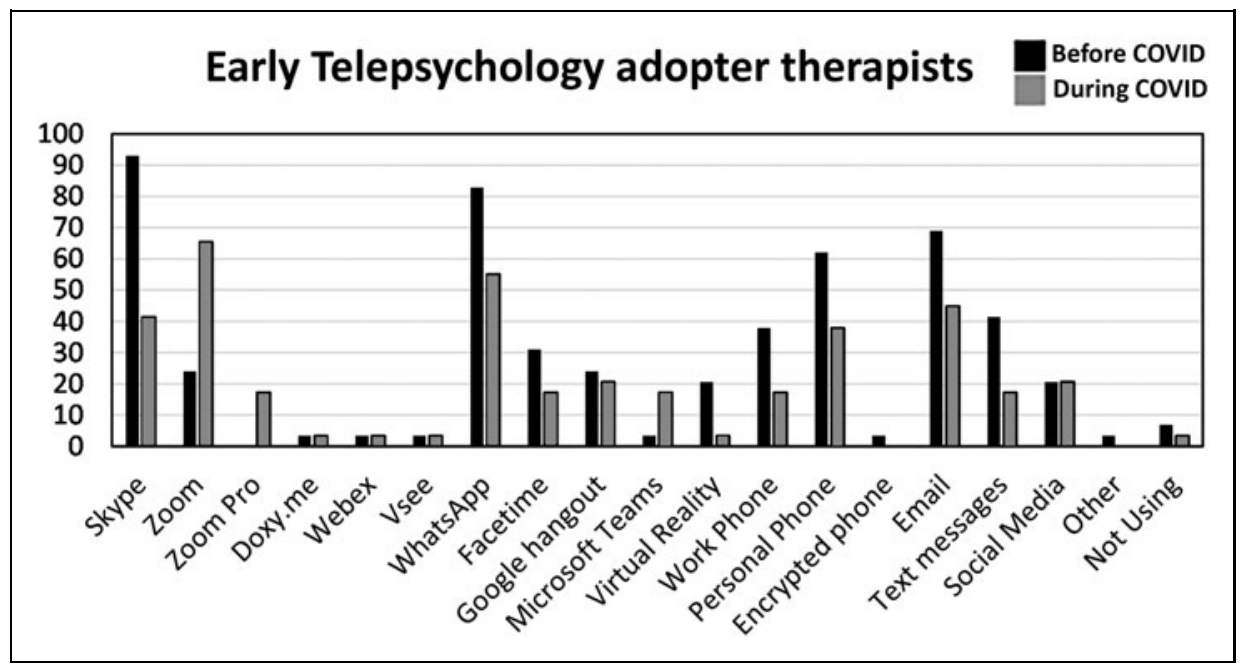

Fig. 3. The most frequent modes of telecommunication used during teletherapy (for early adopters).

\section{NUMBER OF HOURS PER WEEK USING TELEPSYCHOLOGY}

On average, overall (i.e., $N=50$ ), the number of hours per week the therapist spent using telepsychology increased from $3.09 \mathrm{~h} /$ week $\quad(\mathrm{SD}=7.24) \quad$ to $11.48 \mathrm{~h} /$ week $(\mathrm{SD}=10.83)$ during the pandemic, which was a significant increase $(Z=-4.82, p<0.001)$. As shown in Figure 5, compared with prepandemic, the number of hours therapists spent using telepsychology per week increased significantly for early adopter therapists $(Z=-3.18, p=0.001)$ and also for late adopter therapists $(Z=-3.74, p<0.001)$. 


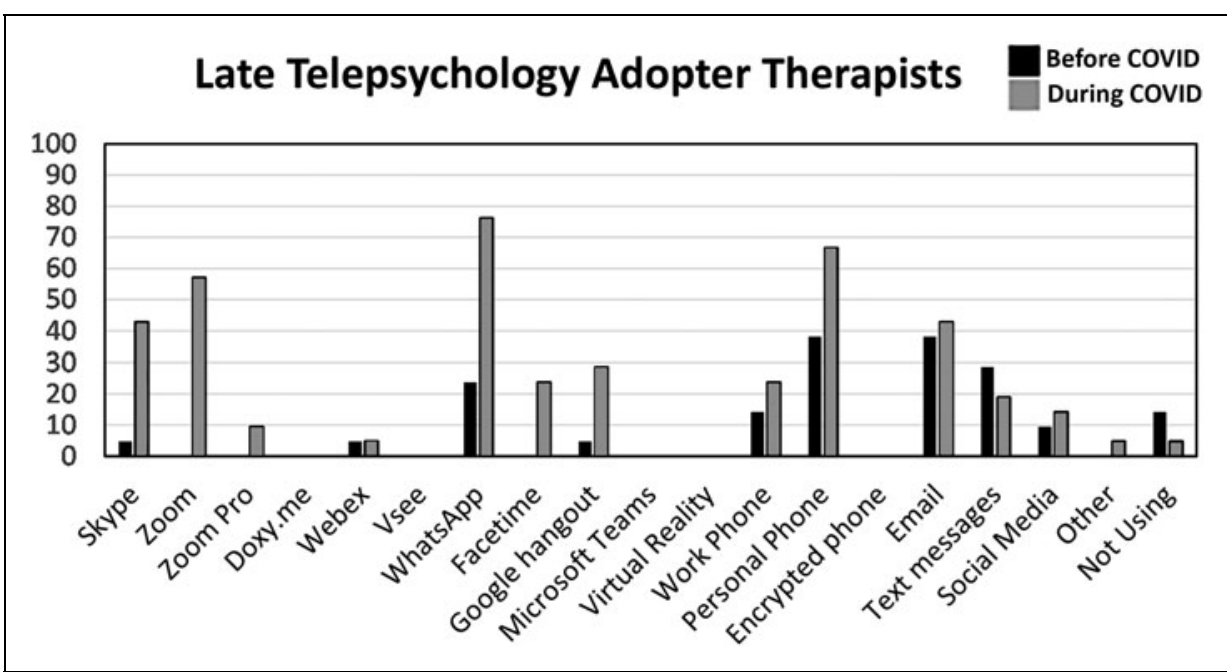

Fig. 4. The most frequent modes of telecommunication used during teletherapy (for late adopters).

\section{ATTITUDES TOWARD TELEPSYCHOLOGY BEFORE VERSUS DURING COVID-19}

The following four Wilcoxon signed-rank related samples comparisons are shown in Figure 6. On a 0 to 10 scale, therapists reported becoming more comfortable using telepsychology during the pandemic (before COVID-19: $M=6.24, \mathrm{SD}=2.60$ vs. during COVID-19: $M=7.60, \mathrm{SD}=1.73 ; Z=-4.26, p<0.001$ ). Therapists also became more confident providing telepsychology without an initial in-person assessment (before COVID-19: $M=5.18, \mathrm{SD}=2.46$ vs. during COVID-19: $M=6.90$, $\mathrm{SD}=2.21$; Wilcoxon signed-rank test with $Z=-4.19, p<0.001$ ).

\section{BURNOUT, MINDFULNESS, AND VR MINDFULNESS}

On our single item 0-10 graphic rating scale measure, Spanish-speaking therapists reported a 16\% increase in feeling burned out during the COVID-19 pandemic (before the pandemic: $M=4.94, \mathrm{SD}=1.98$ vs. during the pandemic: $M=5.92, \mathrm{SD}=2.45 ; Z=-2.71, p=0.007)$.

Therapists reported a small increase in their own personal mindfulness practices during the pandemic (before the pandemic: $M=1.36 \mathrm{~h} /$ week, $\mathrm{SD}=1.69 \mathrm{~h}$ vs. during the pandemic: $2.04 \mathrm{~h} /$ week, $\mathrm{SD}=2.50 \mathrm{~h}$; Wilcoxon signed-rank test with $Z=-2.12, p=0.03)$. The therapists surveyed reported high treatment effectiveness using telepsychology. How effective do you think your telepsychology/telehealth services are? $(0=$ Not effective at all, $10=$ Extremely effective) $(M=7.54, \mathrm{SD}=1.82)$.

Most respondents (84\%) reported that they would use VR mindfulness for their own wellness for at least $1 \mathrm{~h}$ /week. Of those $84 \%$ responding yes, therapists estimated that they would practice $3.83 \mathrm{~h}(\mathrm{SD}=3.83 \mathrm{~h})$ of VR mindfulness per week (Fig. 7).

\section{PROFESSIONAL, ETHICAL, AND LEGAL/REGULATOR ISSUES AND TELEPSYCHOLOGY TRAINING NEEDS}

Therapists' ethical and regulatory concerns about using telepsychology were also assessed (Table 1). Most participants reported concerns regarding security/ confidentiality or Health Insurance Porability and Accountability Act (HIPAA) Compliance and inability to handle emergency situations. Respondents identified a need for increased specialized training and education in the future.

\section{EQUITY ISSUES}

Therapists raised a number of equity issues in the open-ended questions/ comment section of our survey. Many Spanish-speaking patients have lost their jobs and insurance benefits during COVID-19 and cannot continue to pay for therapy sessions, particularly among underserved lower income patients. Many patients do not have the required technology (i.e., tablets, computer) and relied on phone calls. Other inequities therapists mentioned in the comment section include not having privacy for sessions (living in small houses with other family members, making it hard to speak freely to the therapist), not having proper access to technology, noisy neighbors, and difficulty in treating children. One therapist said that telehealth gave them a headache; one therapist reported that new patients were hesitant to sign up in case it was an online scam. Therapists commented that telehealth is very difficult with children, many patients suffering from addiction have abandoned their treatment, and emotion activation techniques are difficult online. Immunosuppressed patients were very grateful to get treatment without risking exposure to the virus, but some hospitals wanted in-person treatments to continue despite the dangers. One therapist said that they are treating patients who live further away than usual. Another therapist mentioned the lack for a legal framework for recording online sessions.

\section{Discussion}

The current study describes the implementation of telehealth and its impact on treatment delivery among non-U.S. Spanish-speaking therapists before and during the COVID19 pandemic. There are several noteworthy outcomes from this study. For one, most Spanish-speaking therapists who responded to the current Spanish version of the survey were already using telehealth before the COVID-19 pandemic 


\section{SAMPAIO ET AL.}

\section{Table 1. Therapists' Ethical and Regulatory Concerns About Using Telepsychology and Telepsychology Training Needs \\ YES, UNSURE, NO, OTHER \\ $\%$ \\ $\%$ \\ $\%$

Survey on professional, ethical, and legal regulatory issues on telehealth training and practice $(N=50)$

\begin{tabular}{|c|c|c|c|}
\hline Does your malpractice carrier cover you for delivering telehealth services? & 12 & 46 & 42 \\
\hline Do you think it is ethical for licensed mental health professionals to deliver services online or via other telehealth technologies? & 92 & 0 & 8 \\
\hline $\begin{array}{l}\text { Do you believe it is legal to practice over state lines or national borders using telecommunications technology if you call } \\
\text { yourself a "coach"? }\end{array}$ & 30 & 36 & 34 \\
\hline $\begin{array}{l}\text { Do you think it is legal for licensed professionals to provide services online to someone who is located in a state } \\
\text { or country in which you are NOT licensed? }\end{array}$ & 28 & 34 & 42 \\
\hline Do you think mental health practitioners should undergo any training about: [The technical issues of telehealth] & 94 & 0 & 6 \\
\hline $\begin{array}{l}\text { Do you think mental health practitioners should undergo any training about: [Clinical, legal, and/or ethical issues } \\
\text { in telehealth] }\end{array}$ & 98 & & 2 \\
\hline $\begin{array}{l}\text { Do you think the average mental health professional can effectively screen for "at risk" clients (dangerous to self } \\
\text { or others) using telehealth technology? }\end{array}$ & 54 & 28 & 18 \\
\hline $\begin{array}{l}\text { Are you aware of any state or federal law/s or regulation/s that govern the delivery of counseling services provided } \\
\text { online or via other telehealth technologies? }\end{array}$ & 30 & 14 & 56 \\
\hline $\begin{array}{l}\text { Are you certified to work with telepsychology/telehealth? (Please remember that this survey is anonymous and that most } \\
\text { places do not require certification in telepsychology/telehealth to practice it) }\end{array}$ & 30 & - & 70 \\
\hline Did your Undergraduate or Graduate University program offer telepsychology/telehealth specific classes or content? & 16 & 6 & 78 \\
\hline $\begin{array}{l}\text { Do you think that it would be helpful for professionals to have training on telepsychology/telehealth as a part of their } \\
\text { professional Undergraduate university program or Graduate University program? }\end{array}$ & 96 & - & 4 \\
\hline
\end{tabular}

What are your concerns about telehealth? (select all that apply)

\begin{tabular}{|c|c|c|c|}
\hline Security/confidentiality or HIPAA compliance & 62 & - & 38 \\
\hline Equipment costs & 10 & - & 90 \\
\hline Licensure issues & 42 & - & 58 \\
\hline Lack of personal training or education in this area & 40 & - & 60 \\
\hline Lack of direction from my professional association & 12 & - & 88 \\
\hline Lack of available education or training programs in this area & 34 & - & 66 \\
\hline Lack of supporting research & 26 & - & 74 \\
\hline Inability to handle emergency situations & 36 & - & 64 \\
\hline Tried online therapy practice and decided it was not for me & 0 & - & 100 \\
\hline Don't know how to get started with online practice & 2 & - & 98 \\
\hline Don't understand issues involved with online practice & 4 & - & 96 \\
\hline Don't have any concerns? & 12 & - & 88 \\
\hline Have you been volunteering your services online to help people through the novel-coronavirus pandemic? & 24 & - & 76 \\
\hline
\end{tabular}

HIPAA, Health Insurance Porability and Accountability Act.

began. Both "early" and "late" adopters reported a large and statistically significant increase in the number of hours of telepsychology per week during COVID-19. Therapists reported becoming significantly more comfortable using telepsychology during the pandemic, and they became more confident providing telepsychology without an initial inperson assessment, but more burned out. Nearly all (98\%) Spanish-speaking respondents recommended increasing training for therapists in college and just in time training. Finally, there was a small but significant increase in 


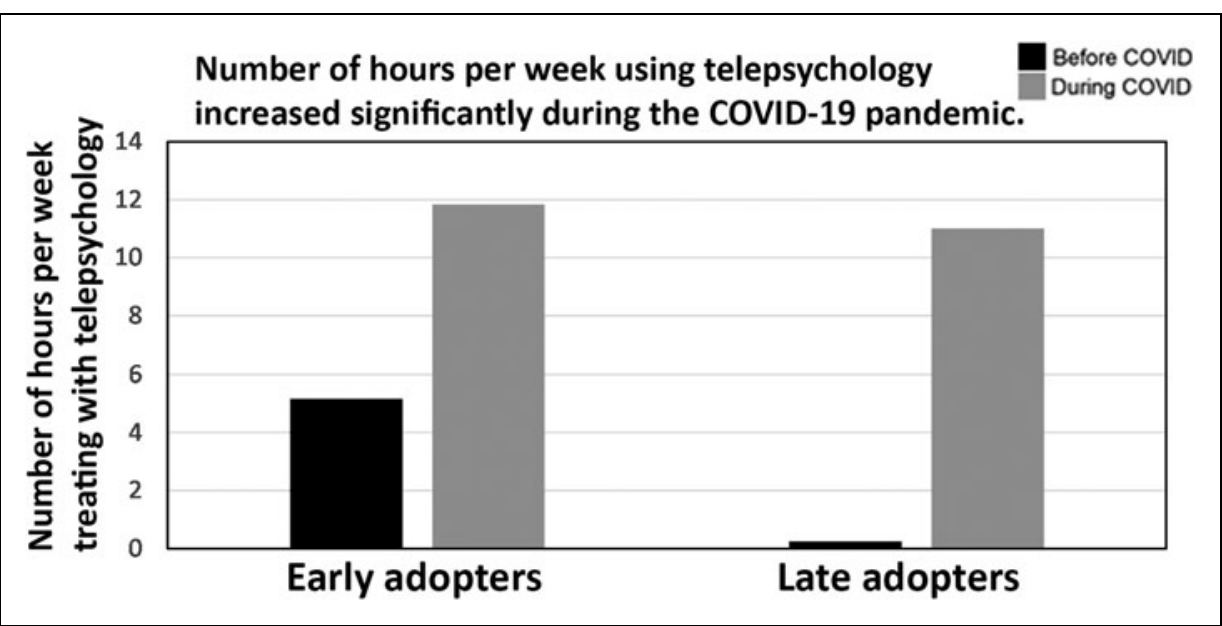

Fig. 5. Number of hours per week the therapist used telepsychology.
Telehealth COVID-19 report provides policy recommendations for future expansion of telehealth access to underserved Spanish-speaking Latino patients (https://latino.ucla.edu/wpcontent/uploads/2020/05/TelehealthCOVID-19-Report.pdf).

There are disparities in telemedicine access for Spanish-speaking patients during the COVID-19 crisis, ${ }^{20}$ but getting more contact information from Spanish-speaking patients (e.g., e-mail and medical portal activation) may increase their access to telemedicine. Taking that idea one step further, we recommend showing patients how to set up their cell phones and/or home

the number of hours per week the therapists practiced mindfulness. We describe the implications of these findings below.

The Spanish-speaking therapists mentioned a number of inequities in the open-ended comment section. The rapid adoption of technology to deliver therapy has spurred growing pains for Spanish-speaking therapists and their clients, and more research is needed to better understand and improve the adoption of these technologies with underserved populations. Therapist respondents identified a nearly unanimous need for increased specialized training and education in the future. Based on these and other results, updating/reducing regulatory restrictions on telehealth is also recommended. The following

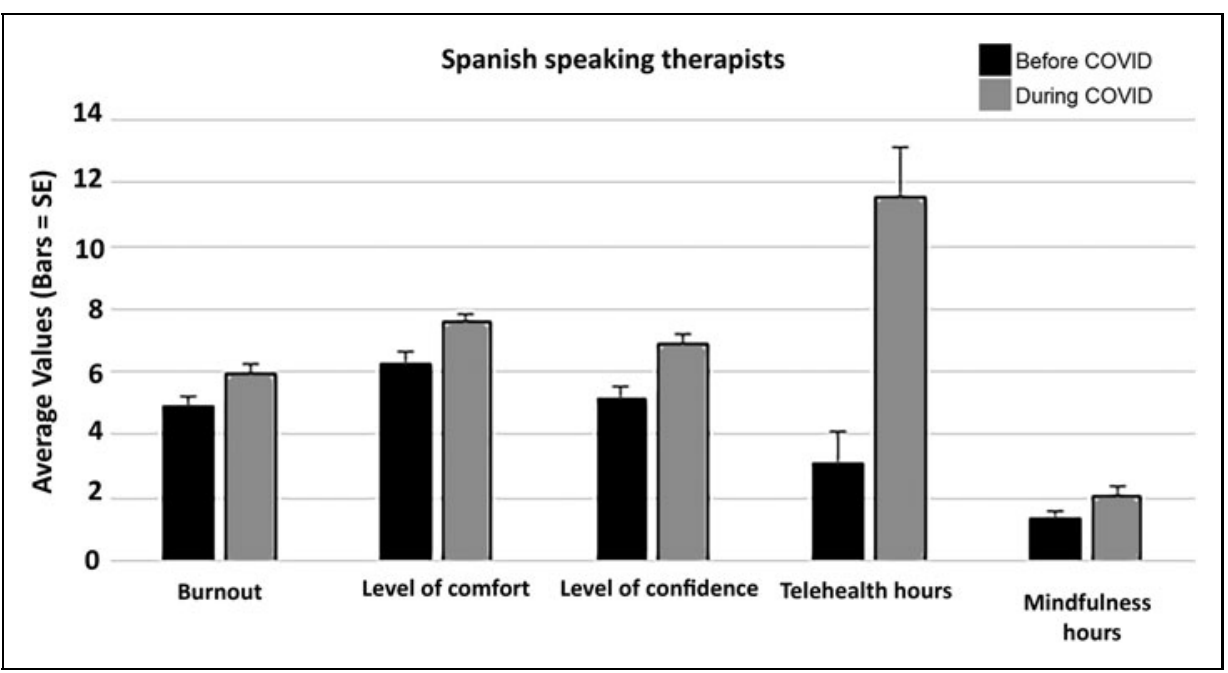

Fig. 6. Wilcoxon signed-rank paired comparisons of therapists' comfort, confidence, burnout, and use of mindfulness, and percentage of therapists using telepsychology before versus during the pandemic. computer and/or VR system and practicing telehealth communications with patients who are currently only "in person," to make it easier for them to use telehealth. If they have a laptop or cell phone, they can bring those devices to the clinic to practice using telepsychology technologies (once patients and therapists get vaccinated and in-person visits become safer).

\section{FUTURE DIRECTIONS: IMMERSIVE VR TELEPSYCHOLOGY}

The current results show a large increase in therapists' use of computer-augmented clinical therapy in Spanish-speaking therapists during COVID-19. The current survey suggests that immersive VR technology is beginning to be adopted into mainstream clinical practice in Spain and Latin America. For example, in the current survey, before the COVID-19 pandemic, 20\% of the early adopter therapists indicated that they had used VR technology to help treat their patients. Furthermore, a large number of respondents (84\%) expressed interest in trying immersive VR mindfulness to reduce their own stress. These results provide early indications that Spanish-speaking therapists are beginning to use VR to help their patients, a trend we predict will increase substantially in the future, as the use of VR becomes more mainstream and widely adopted by the general public, for example, for entertainment. ${ }^{21}$ 


\section{SAMPAIO ET AL.}

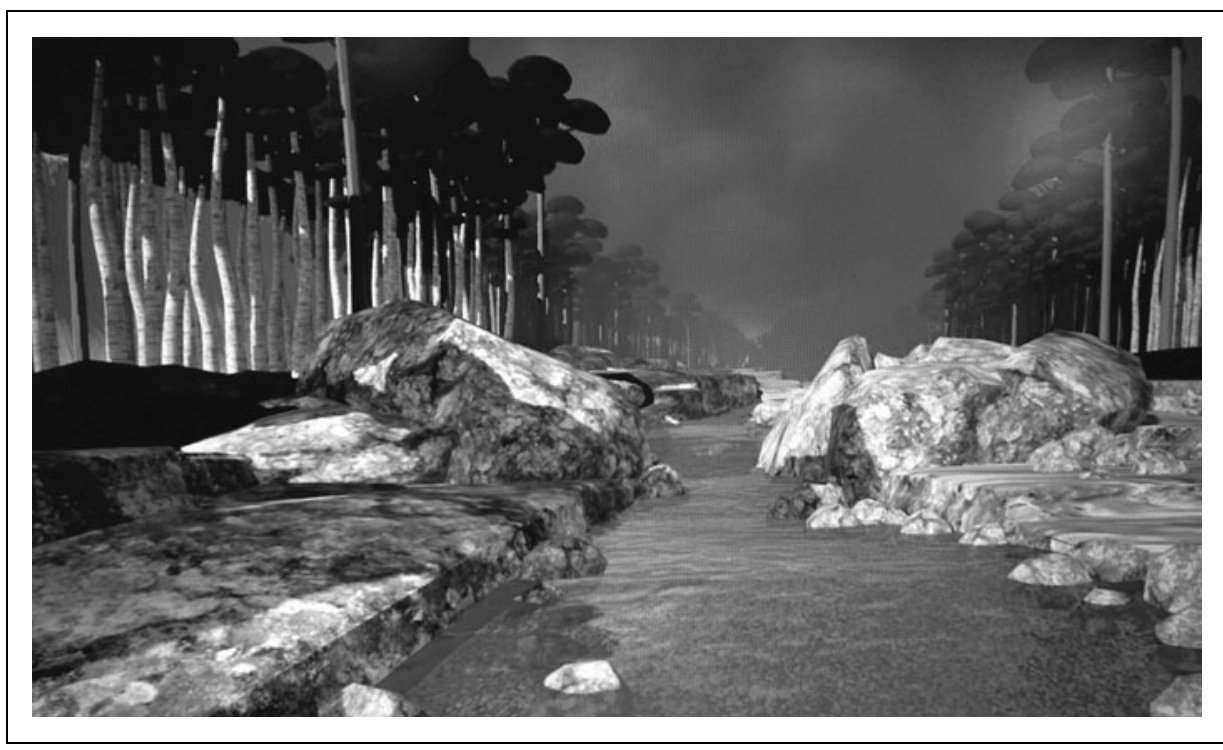

Fig. 7. Mindfulness RiverWorld, image by bigenvironments.com, copyright Hunter Hoffman, www.vrpain.com

Most therapeutic immersive VR experiences to date have been single user, with the patient receiving VR in the therapist's office while getting therapeutic guidance from the therapist who was traditionally also in the same room with the patient. During VR therapy (e.g., exposure therapy for spider phobia), patients put on a VR helmet and interact with objects in the virtual world (e.g., a virtual spider), while getting instructions and encouragement from their therapist. But, to date, most VR therapy patients have been alone in the single player virtual world. The therapist was not wearing a VR helmet. The therapist could watch what the patient was seeing and doing, via a two-dimensional computer monitor, but the therapist was in the real world, the patient was in the virtual world. In the future, with networked immersive VR becoming more widely available, secure, and inexpensive, therapists and patients in different physical locations can in theory "meet" and "be there together" in a shared computer-generated world designed for multiuser therapy sessions, including one-onone sessions and group therapy sessions. ${ }^{22}$ Future studies may explore the use of new multimodal clinical telepsychology therapy treatment approaches that include networked shared immersive VR technology.

\section{LIMITATIONS}

The current study has several limitations to consider when interpreting these results. For one, this survey asked participants to retrospectively assess many items pre- and periCOVID. Future studies should integrate multiple time points to assess change in desired variables. Most survey partici- pants were identified as female, which may or may not accurately reflect the demographics, attitudes, and behaviors of Spanish-speaking therapists. The current study used an original single-item burnout rating that has not yet been validated. Although other studies have shown single-item burnout ratings to be valid, ${ }^{23}$ future studies should also include traditional measures of burnout in which the validity and reliability have already been established. Although survey questionnaires are a valuable exploratory descriptive method, the use of more refined interviews and self-report measures as well as randomized controlled clinical and laboratory follow-up studies are needed to better understand the research topics addressed in the current study, and to test new treatments for reducing the psychological consequences of the COVID-19 pandemic. Sampaio et al. ${ }^{1}$ evaluated for the first time the psychometrics of the current survey instrument. Based on their exploratory factor analyses, they recommended that for future studies, all items and response choices of surveys should use interval or ratio scales, or at the very least, ordinal, avoiding nominal categories and particularly avoiding multiple choices questions. $^{24}$

\section{Conclusions}

In conclusion, this online survey provided insight into the changes in telepsychology adoption and changes in Spanish-speaking therapists' attitudes and behavior toward using telepsychology to conduct therapy sessions in response to the COVID-19 pandemic. The current results indicate that there has been a significant increase in the use of telepsychology to conduct psychotherapy during the COVID-19 pandemic by mental health professionals in Spain and Latin American countries and now that they are using telepsychology regularly, most therapists surveyed now feel more comfortable using telepsychology with their patients, even without an initial "in person" assessment. However, a number of therapists still had ethical, training, and personal concerns regarding the use of telepsychology, and gaps in therapists' knowledge on these topics were evident, indicating a strong need for increased telepsychology training for therapists in the future. The current study also identified a significant increase in therapists' self-ratings of burnout 
during COVID-19, suggesting that Spanish-speaking therapists could benefit from additional training on their own stress coping skills during a crisis (e.g., practicing mindfulness while in a computer-generated virtual world, ${ }^{13-16,25,26}$ and a number of other coping strategies could also be valuable). Finally, COVID-19 will likely increase the number of therapists needed in Spain and Latin American countries, and worldwide, ${ }^{27}$ to help mitigate the long-term psychological and economic consequences of the pandemic. $^{28,29}$ If there is a growing gap between the number of patients and the availability of highly trained therapists, and economic considerations during a damaged economy, computer-augmented therapy may be a cost-reducing way to help increase dissemination of effective psychological stress reduction treatments to underserved patient populations. Research and development of "COVID-19 therapy" is needed (e.g., improving the user experience to reduce dropouts $^{30}$ ).

\section{Disclosure Statement}

M.S. is a co-owner of https://www. mindovermattersinstitute .com/mariana-sampaio-mhc-msw-cpp. C.W. receives consulting fees from Mindstrong Health and Lyra Health. H.G.H. is a scientific advisor to BehaVR.com.

\section{Funding Information}

Mariana and Sampaio worked on this project pro bono. During preparation of this article, Hunter G. Hoffman was supported by charitable funds from the Mayday Fund and by NIH R01 GM042725 to Dave Patterson; Maria Vicenta Navarro Haro was supported by Gobierno de Aragón (Group reference: S31_20D) and by Feder 2014-2020 "Construyendo Europa desde Aragón”; and Bruno De Sousa is a professor at the University of Coimbra.

\section{REFERENCES}

1. Sampaio M, Haro MVN, De Sousa B, Melo WV, Hoffman HG. Therapists make the switch to telepsychology to safely continue treating their patients during the COVID-19 pandemic. Virtual reality telepsychology may be next. Front Virtual Real 2021;1:576421.

2. Pierce BS, Perrin PB, Tyler CM, McKee GB, Watson JD. The COVID-19 telepsychology revolution: A national study of pandemic-based changes in U.S. mental health care delivery. Am Psychol 2021;76:14-25.

3. Eccleston $C_{\text {, Fisher }}$, Howard RF, et al. Delivering transformative action in paediatric pain: A Lancet Child \& Adolescent Health Commission. Lancet Child Adolesc Health 2021;5:47-87.

4. Tauben DJ, Langford DJ, Sturgeon JA, et al. Optimizing telehealth pain care after COVID-19. Pain 2020;161:2437-2445.
5. Hincapié MA, Gallego JC, Gempeler A, Piñeros JA, Nasner D, Escobar MF. Implementation and usefulness of telemedicine during the COVID-19 pandemic: A scoping review. J Prim Care Community Health 2020;11: 2150132720980612.

6. Boeldt $D$, McMahon $E$, McFaul M, Greenleaf W. Using virtual reality exposure therapy to enhance treatment of anxiety disorders: Identifying areas of clinical adoption and potential obstacles. Front Psychiatry 2019;25; 10:773.

7. Difede J, Cukor J, Wyka K, et al. D-cycloserine augmentation of exposure therapy for post-traumatic stress disorder: A pilot randomized clinical trial. Neuropsychopharmacology 2014;39:1052-1058.

8. Difede J, Hoffman HG. Virtual reality exposure therapy for world trade center post-traumatic stress disorder: A case report. Cyberpsychol Behav 2002;5: 529-535.

9. García-Palacios A, Hoffman H, Carlin A, Furness TA, Botella C. Virtual reality in the treatment of spider phobia: A controlled study. Behav Res Ther 2002;40: 983-993.

10. Hoffman HG. Virtual-reality therapy. Sci Am 2004;291:58-65.

11. Rizzo A, Bouchard $S$, eds. Virtual reality for psychological and neurocognitive interventions. New York: Springer, 2019.

12. Rizzo A, Shilling R. Clinical Virtual Reality tools to advance the prevention, assessment, and treatment of PTSD. Eur J Psychotraumatol 2017;8: 1414560.

13. Navarro-Haro MV, Hoffman HG, Garcia-Palacios A, et al. The use of virtual reality to facilitate mindfulness skills training in dialectical behavioral therapy for borderline personality disorder: A case study. Front Psychol 2016;7:1573.

14. Gomez J, Hoffman HG, Bistricky SL, et al. The use of virtual reality facilitates Dialectical Behavior Therapy ${ }^{\circledR}$ "Observing Sounds and Visuals" mindfulness skills training exercises for a Latino patient with severe burns: A case study. Front Psychol 2017;8:1611.

15. Flores $A$, Linehan MM, Todd SR, Hoffman HG. The use of virtual reality to facilitate Mindfulness skills training in Dialectical behavioral therapy for spinal cord injury: A case study. Front Psychol 2018;9:531.

16. Navarro-Haro MV, Modrego-Alarcón M, Hoffman HG et al. Evaluation of a mindfulness-based intervention with and without Virtual Reality Dialectical Behavior Therapy ${ }^{\circledR}$ mindfulness skills training for the treatment of generalized anxiety disorder in primary care: A pilot study. Front Psychol 2019;10:55.

17. Glueckauf RL, Maheu MM, Drude KP. Survey of psychologists' telebehavioral health practices: Technology use, ethical issues, and training needs. Profess Psychol Res Pract 2018;49:205-219.

18. Freudenberger HJ. Staff burnout. J Soc Issues 1974;30:159-165.

19. IBM Corp. Released 2019. IBM SPSS Statistics for Windows, Version 26.0. Armonk, NY: IBM Corp., 2019.

20. Blundell AR, Kroshinsky D, Hawryluk EB, Das S. Disparities in telemedicine access for Spanish-speaking patients during the COVID-19 crisis. Pediatr Dermatol 2020. [Epub ahead of print]; DOI: 10.1111/pde. 14489.

21. Bailenson JN. Experience on demand: What virtual reality is, how it works, and what it can do. New York: W.W. Norton, 2018.

22. Dilgul M, Hickling LM, Antonie D, Priebe S, Bird VJ. Virtual Reality Group therapy for the treatment of depression: A qualitative study on stakeholder perspectives. Front Virtual Real 2021;1: 609545

23. Dolan ED, Mohr $D$, Lempa M. Using a single item to measure burnout in primary care staff: A psychometric evaluation. J Gen Intern Med 2015;30; 582-587.

24. McDonald RP. Test theory: A unified treatment. Mahwah, NJ: Erlbaum, 1999. 
25. Navarro-Haro MV, López-del-Hoyo Y, Campos D, et al. Meditation experts try Virtual Reality Mindfulness: A pilot study evaluation of the feasibility and acceptability of Virtual Reality to facilitate mindfulness practice in people attending a Mindfulness conference. PLoS One 2017;12:e0187777.

26. Navarro-Haro MV, Harned MS, Korslund KE, et al. Predictors of adoption and reach following Dialectical Behavior Therapy Intensive Training ${ }^{\mathrm{TM}}$. Community Ment Health J 2019;55:100-111.

27. World Health Organization. Mental Health and Psychosocial Considerations During COVID-19 Outbreak. 2020. Available at https://www.who.int/publications/ i/item/WHO-2019-nCoV-MentalHealth-2020. (last accessed June 17, 2021).

28. Pfefferbaum B, North CS. Mental health and the Covid-19 pandemic. N Eng/ J Med 2020;6:510-512.

29. Singh S, Roy D, Sinha K, Parveen S, Sharma G, Joshi G. Impact of COVID-19 and lockdown on mental health of children and adolescents: A narrative review with recommendations. Psychiatry Res 2020;293:113429.

30. Wilks CR, Yin Q, Zuromski KL. User experience affects dropout from internetdelivered dialectical behavior therapy. Telemed J E Health 2020;26: 794-797.
Address correspondence to:

Hunter G. Hoffman, PhD

Human Photonics Lab

Virtual Reality Research Center

University of Washington

Box 352142

Seattle, WA 98195-2180

USA

E-mails: hunthoff9@gmail.com; hunthoff@uw.edu

Received: February 28, 2021

Revised: April 1, 2021

Accepted: April 1, 2021

Online Publication Date: June 29, 2021 